\title{
Methodologies for the Study of Food Systems by Environmental Scanning Electron Microscopy (ESEM)
}

\author{
D.J. Stokes, B.L. Thiel \& A.M. Donald
}

Polymers \& Colloids Group, University of Cambridge, Department of Physics, Cavendish Laboratory, Madingley Road, Cambridge, CB3 OHE, UK

ESEM can be used to image insulating and/or moist specimens without the need for the removal of volatile components or the application of a conductive coating. This ability significantly increases the range of experiments and observations that can be performed at the high resolution of electron microscopy, yielding new perspectives on microstructure to complement information derived from other techniques. Such a technological advance has particularly important implications for the study of soft matter, complex fluids and biological specimens.

Foods fall into all three of the above categories: the chemical and physical components of a food, together with processing conditions, result in a microstructure that determines qualities such as texture, flavor and appearance. An understanding of food structure obtained by microscopic techniques, in conjunction with knowledge of the macroscopic properties, offers ways to improve existing products and design new ones. Since these materials often contain a number of components that may not survive traditional EM preparation techniques, food systems are an excellent example of where ESEM can be used to probe the structure-property relationships of the native material, and specific applications in this field are outlined elsewhere [1].

Maximising the potential of ESEM requires the development of new techniques and methodologies, in order to control the stability of food specimens in situ whilst achieving good quality images and reproducible results. Consideration must be given to the chamber water vapor conditions, the state of any water present in the specimen (e.g. bound or containing dissolved ions), the design of apparatus/experiments and image interpretation, particularly where multiphase, multi-component food systems are involved.

The range of specimen temperatures and partial pressures of water vapor that can be used in ESEM is a crucial aspect of specimen stability and affords the opportunity to manipulate the state of the specimen. A common misconception is that thermodynamic equilibrium conditions require an environment approaching 100\% relative humidity (RH), as depicted in Figure 1. However, there are several factors that should be taken into account when choosing operating parameters for a given RH. Briefly, these include the effects of admitting water vapor molecules into the specimen chamber from a source at room temperature [2], the kinetics of water mass loss and, most importantly, the activity of water (or osmotic pressure) in the specimen itself. Taken together, these factors can significantly reduce the partial pressure of water required for stabilising hydrated or liquid specimens. For example, if the distribution of dispersed phases in a complex fluid is to be studied, then typical conditions tend to be $3^{\circ} \mathrm{C}$ at a vapor pressure of 4.5 torr [3], [4]. With reference to Figure 1, it can be seen that this is somewhat below $100 \% \mathrm{RH}$, and yet the specimen remains stable. Results such as this suggest that equilibrium conditions for a water-containing specimen deviate from those of pure water by as much as $20-25 \%$. A major advantage of this flexibility in operating parameters is that conditions can be chosen for more favorable image formation. 
In more sophisticated studies, water vapor can be made to evaporate or condense onto the specimen, permitting observation of moisture-dependent dynamic structural changes. Further, if this is carried out at sub-zero temperatures, it is possible to manipulate crystalline (frozen) phases in food specimens possessing low glass transition temperatures, such as ice cream, by controlled sublimation of ice. Again, in accordance with Figure 1, a small partial pressure of water vapor is required. Depending on gaseous detector type, it becomes appropriate to use an additional imaging gas when using such small partial pressures of water vapor, in order to provide a mechanism for gaseous signal amplification. Such a gas should be inert, non-toxic, relatively inexpensive and possess a vapour pressure low enough to avoid condensation at very low temperatures. A good example of such a gas is nitrous oxide, which combines good amplification/charge compensation properties and signal-tonoise ratio with a vapor pressure of approximately 0.1 torr at $-150^{\circ} \mathrm{C}$ [5].

Other relevant topics include secondary electron contrast arising from differences in electronic structure between phases [3] and the use of dynamic contrast phenomena as an aid to identifying the presence of lipids [4], as well as the issue of image resolution to be expected as a result of electron interactions with soft, low atomic number materials such as foods.

\section{References}

1. B.L. Thiel, D.J. Stokes \& A.M. Donald, elsewhere in these proceedings.

2. R. Cameron \& A. Donald, J. Microscopy, 173(3) (1994) p. 227-237.

3. D.J. Stokes, B.L. Thiel \& A.M. Donald, Langmuir, 14(16) (1998) p. 4402-4408.

4. D.J Stokes, B.L. Thiel \& A.M. Donald, Scanning, 22(6) (2000) p. 357-365.

5. A. Fletcher, B. Thiel \& A. Donald, J. Phys. D: Appl. Phys., 30 (1997) p. 2249-2257.

6. Funding from the Royal Society, BBSRC, Unilever, FEI Company \& the Sir Isaac Newton Trust are gratefully acknowledged.

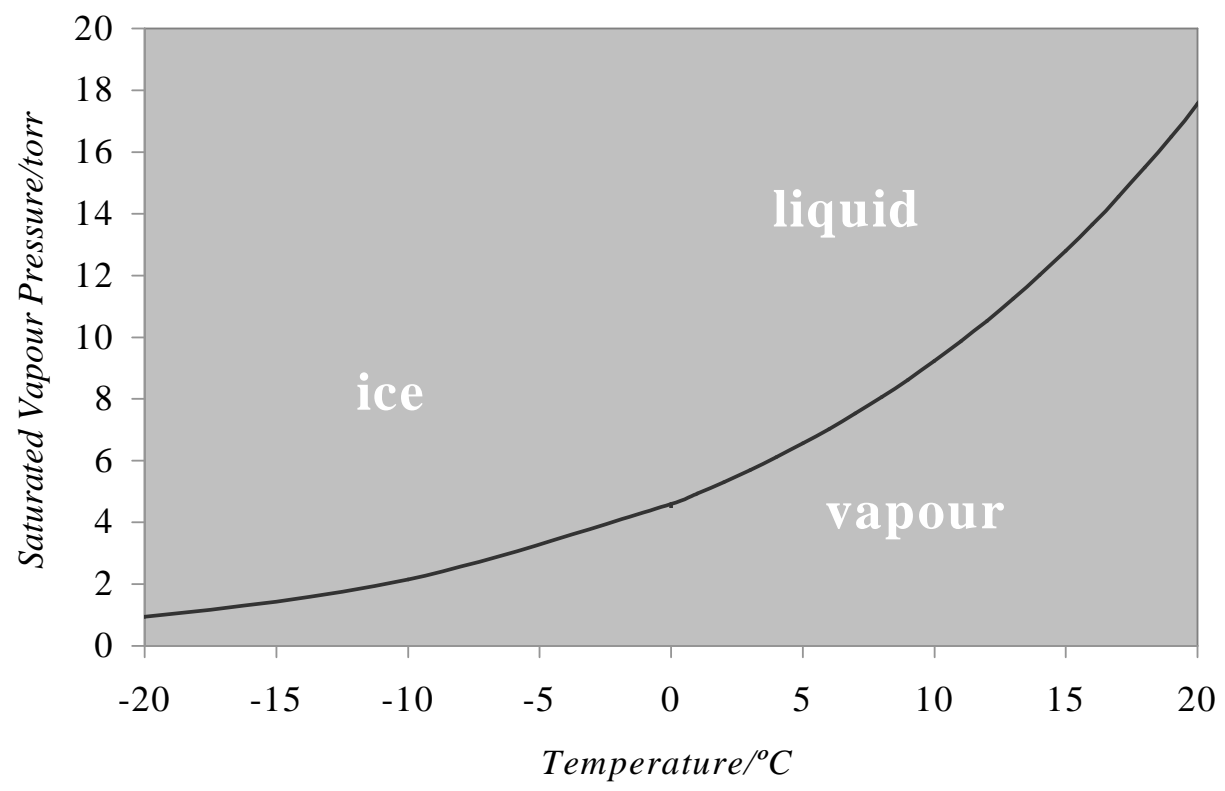

Figure 1. Saturated vapor pressure curve of pure water, indicating thermodynamic phase equilibria. 